\title{
Energy levels in muonic helium
}

\author{
A.V. Eskin ${ }^{1, *}$, V.I. Korobov ${ }^{1,2}$, A.P. Martynenko ${ }^{1}$, and V.V. Sorokin ${ }^{1}$ \\ ${ }^{1}$ Samara National Research University, Department of General and Theoretical Physics, 443086 \\ Samara, Russia \\ ${ }^{2}$ Joint Institute for Nuclear Research, BLTP, 141980 Dubna, Russia
}

\begin{abstract}
The energy spectrum of bound states and hyperfine structure of muonic helium is calculated on the basis of stochastic variational method. The basis wave functions of muonic helium are taken in the Gaussian form. The matrix elements of the Hamiltonian are calculated analytically. For numerical calculation a computer code is written in the MATLAB system. As a result, numerical values of bound state energies and hyperfine structure are obtained. We calculate also correction to the structure of the nucleus, vacuum polarization and relativistic correction.
\end{abstract}

\section{Introduction}

The muonic helium atoms $\left(\mu e_{2}^{4} \mathrm{He}\right),\left(\mu e_{2}^{3} \mathrm{He}\right)$ are the simple three-body atomic systems composed of negative muon, electron and nucleus. The Coulomb interaction between particles leads to the formation of bound states. The interaction between magnetic moments of the muon, electron and helion gives the hyperfine structure (HFS) of the energy levels. The investigation of energy spectrum of this atom is important for the check of quantum electrodynamics for three-particle bound state. The ground state hyperfine splitting was measured many years ago with sufficiently high accuracy [1]:

$$
\Delta v_{\text {exp }}^{\text {hfs }}=4465.004(29) \mathrm{MHz} \text {. }
$$

Hydrogen-like bound-states were investigated in QED with very high accuracy [2-4]. Contrary to two-particle bound states three-particle systems were studied on the basis of perturbation theory (PT) and variational approach with smaller accuracy. There are several calculations devoted to HFS of the excited state $1 s_{1 / 2}^{(e)} 2 s_{1 / 2}^{(\mu)}$ in muonic helium atom [5-6]. In these papers different order corrections to HFS were studied by perturbation theory and on the basis of variational method. The variational method gives high numerical accuracy of the calculation what was demonstrated in [7-10].

Bound particles in muonic helium have different masses $m_{e} \ll m_{\mu} \ll m_{\alpha}$. As a result the muon and $\alpha$-particle (or helion) compose the pseudonucleus $\left(\mu_{2}^{3,4} \mathrm{He}\right)^{+}$and muonic helium atom looks as a two particle system in first approximation. The existence of such mass hierarchy enables to formulate a perturbation theory for the calculation of the energy levels. The perturbation theory approach to the investigation of hyperfine structure of muonic helium based on nonrelativistic Schrödinger equation was developed in [11-12].

\footnotetext{
* Corresponding author: EskinAlexey1992@gmail.com
} 
It should be noted that new precise measurement of hyperfine structure of muonic helium is planned at J-PARC MUSE [13] because high intensity pulsed negative muon beam gives an opportunity to improve the result (1) by two orders of magnitude. Another important energy interval that could be investigated experimentally could be the electron Lamb shift (2P-2S) [14-15]. In this work we study hyperfine structure and the electron Lamb shift in muonic helium atom using the stochastic variational approach [16-18].

\section{General formalism}

The perturbation theory approach to the investigation of the energy structure of muonic helium is based on nonrelativistic Schrödinger equation [11-12]. Using this method we can obtain an estimation of the electron Lamb shift. For this aim we describe the excited electron states $2 \mathrm{~S}$ and $2 \mathrm{P}$ in three-particle bound system $\left(\mu e_{2}^{3,4} \mathrm{He}\right)$ by the Hamiltonian:

$$
\begin{gathered}
H=H_{0}+\Delta H+\Delta H_{r e c}, \quad H_{0}=-\frac{1}{2 M_{\mu}} \nabla_{\mu}^{2}-\frac{1}{2 M_{e}} \nabla_{e}^{2}-\frac{2 \alpha}{x_{\mu}}-\frac{\alpha}{x_{e}}, \\
\Delta H=\frac{\alpha}{x_{\mu e}}-\frac{\alpha}{x_{e}}, \quad \Delta H_{r e c}=-\frac{1}{m_{\alpha}} \nabla_{\mu} \cdot \nabla_{e}
\end{gathered}
$$

where $x_{\mu}$ and $x_{e}$ are the coordinates of the muon and electron relative to the helium nucleus, $M_{e}=m_{e} m_{\alpha} /\left(m_{e}+m_{\alpha}\right), M_{\mu}=m_{\mu} m_{\alpha} /\left(m_{\mu}+m_{\alpha}\right)$ are the reduced masses of subsystems $\left(e_{2}^{3,4} \mathrm{He}\right)^{+}$and $\left(\mu_{2}^{3,4} \mathrm{He}\right)^{+}$. The wave functions of three-particle system can be taken as follows $\left(a_{1}=M_{e} / M_{\mu}\right)$ :

$$
\begin{aligned}
& \psi_{2 S}=\frac{1}{2 \sqrt{2} \pi}\left(W_{\mu} W_{e}\right)^{3 / 2}\left(1-\frac{W_{e} x_{e}}{2}\right) e^{-W_{\mu} x_{\mu}} e^{-\frac{1}{2} W_{e} x_{e}}, \\
& \psi_{2 P}=\frac{1}{2 \sqrt{6 \pi}}\left(W_{\mu} W_{e}\right)^{3 / 2} W_{e} x_{e} e^{-W_{\mu} x_{\mu}} e^{-\frac{1}{2} W_{e} x_{e}}(\boldsymbol{\varepsilon n}),
\end{aligned}
$$

Then the matrix elements of the Hamiltonian $\Delta H$ can be calculated analytically:

$$
\begin{gathered}
\Delta E_{2 S}^{L S}=<\psi_{2 S}|\Delta H| \psi_{2 S}>=-\frac{W_{e} \alpha}{\left(2+a_{1}\right)^{5}}\left[8+a_{1}\left(20+a_{1}\left(12+a_{1}\left(10+a_{1}\right)\right)\right)\right], \\
\Delta E_{2 P}^{L S}=<\psi_{2 P}|\Delta H| \psi_{2 P}>=\frac{4 W_{e} \alpha}{\left(2+a_{1}\right)^{5}}\left[8+a_{1}\left(20+a_{1}\left(20+a_{1}\left(10+a_{1}\right)\right)\right)\right] .
\end{gathered}
$$

As a result in the leading order in $\mathrm{M}_{\mathrm{e}} / \mathrm{M}_{\mu}$ the Lamb shift is equal to

$$
\Delta E(2 P-2 S)=\frac{\alpha^{2} M_{e}^{3}}{16 M_{\mu}^{2}}=9.619 \mathrm{GHz}
$$

To increase the accuracy of the calculation we can take into account different corrections by perturbation theory. Another way for solving this task is related with stochastic variational approach in which the trial function for a system of particles can be written in the following form:

$$
\Psi=\sum_{i=1}^{K} c_{i} \varphi\left(\mathbf{x}, A_{i}\right),
$$

where $\varphi\left(\mathbf{x}, A_{i}\right)$ is the basic function, $c_{i}$ are linear variational parameters, $\mathbf{x}=\left(x_{1}, \ldots, x_{N-1}\right)$ are the Jacobi coordinates, $A_{i}$ are nonlinear variational parameters, $K$ is the basis size. An upper bound of the ground state energy of the system is given by the lowest eigenvalue of the generalized eigenvalue problem:

$$
\begin{gathered}
H C=E_{K} B C, \\
H_{i j}=<\varphi\left(x_{i}, A_{i}\right)|H| \varphi\left(x_{j}, A_{j}\right)>, \\
B_{i j}=<\varphi\left(x_{i}, A_{i}\right) \mid \varphi\left(x_{j}, A_{j}\right)>.
\end{gathered}
$$

where $H$ is the Hamiltonian of the system, $E_{k}$ is the energy of bound state and $C$ is the eigenvector. Diagonal elements of the $2 \times 2$ dimensional symmetric, positive definite matrix A correspond to nonlinear parameters of a Gaussian expansion, and off-diagonal 
elements connect different relative coordinates representing the correlations between the particles.

In atomic physics the trial function $\varphi\left(x_{i}, A_{i}\right)$ of Hylleraas type or correlated exponential type is often used with success $[7,9,10]$. This function has the exponential form expressed in terms of the interparticle-distance coordinates. Instead of the exponential function let us consider its Gaussian analogue [8].

The trial wave function of the mesomolecules with nonzero angular momentum is written as the superposition of Gaussian basis wave functions

$$
\varphi_{L S}(\mathbf{x}, A)=e^{-\frac{1}{2} \tilde{\mathbf{x}} A \mathbf{x}} \theta_{L}(\mathbf{x}) \chi_{S M_{S}}, \quad \theta_{L}(\boldsymbol{x})=\left[\left[\left[\mathrm{Y}_{\mathrm{l}_{1}}\left(x_{1}\right) \mathrm{Y}_{\mathrm{l}_{2}}\left(x_{2}\right)\right]_{L_{12}} \mathrm{Y}_{\mathrm{l}_{3}}\left(x_{3}\right)\right]_{L_{123}} \ldots\right]_{L M}
$$

where $\mathrm{Y}_{\mathrm{l}_{\mathrm{m}}}(\mathbf{x})=\mathrm{r}^{\mathrm{l}} \mathrm{Y}_{\mathrm{lm}}(\mathbf{x}), l$ and $m$ is orbital and magnetic quantum numbers respectively and $\chi_{S M_{S}}$ is the spin wave function. In the case of three nonidentical particles the wave function for ground state has a form:

$$
\varphi_{00}(\boldsymbol{\rho}, \lambda, A)=e^{-\frac{1}{2}\left[A_{11} \boldsymbol{\rho}^{2}+A_{22} \lambda^{2}+2 A_{12}(\boldsymbol{\rho} \cdot \lambda)\right]},
$$

where $\rho$ and $\lambda$ are the Jacobi coordinates of three particles which are related with the particle radius vectors:

$$
\mathbf{r}_{12}=\mathbf{r}_{1}-\mathbf{r}_{2}=\boldsymbol{\rho}, \quad \mathbf{r}_{13}=\mathbf{r}_{1}-\mathbf{r}_{3}=\boldsymbol{\lambda}+\frac{m_{2}}{m_{12}} \boldsymbol{\rho}, \mathbf{r}_{23}=\mathbf{r}_{2}-\mathbf{r}_{3}=\boldsymbol{\lambda}-\frac{m_{1}}{m_{12}} \boldsymbol{\rho},
$$

where $m_{1}, m_{2}$ and $m_{3}$ are the helium, muon and electron masses, $m_{12}=m_{1}+m_{2}, \mathbf{r}_{12}$ is the radius vector between helium nucleus and muon, $\mathbf{r}_{13}$ is the radius vector between helium nucleus and electron, $\mathbf{r}_{23}$ is the radius vector between muon and electron. In the case of P-states $(\mathrm{L}=1)$, there are three versions of the wave function representation:

$$
\begin{aligned}
& \varphi_{10}(\boldsymbol{\rho}, \boldsymbol{\lambda}, A)=e^{-\frac{1}{2}\left[A_{11} \boldsymbol{\rho}^{2}+A_{22} \lambda^{2}+2 A_{12}(\boldsymbol{\rho} \cdot \lambda)\right]}(\varepsilon \boldsymbol{\rho}), \\
& \varphi_{01}(\boldsymbol{\rho}, \boldsymbol{\lambda}, A)=e^{-\frac{1}{2}\left[A_{11} \boldsymbol{\rho}^{2}+A_{22} \lambda^{2}+2 A_{12}(\boldsymbol{\rho} \cdot \lambda)\right]}(\varepsilon \boldsymbol{\lambda}),
\end{aligned}
$$

where the tensor representation for the angular part of the wave function is used. The basis wave function of the P-state is defined by the sum of functions in (13)-(14) in the form $\varphi(\boldsymbol{\rho}, \lambda)=\mathrm{c}_{1} \varphi_{10}(\boldsymbol{\rho}, \lambda)+\mathrm{c}_{2} \varphi_{01}(\boldsymbol{\rho}, \lambda)$.

For numerical calculation, a program was written in the MATLAB system for solving the three-particle Coulomb problem in the framework of the stochastic variational method. The program from [8] in the Fortran language was taken as the basis. With the proper selection of trial functions, this method can give very close to real values. In particular, if the type of the test function is accurate to the choice of parameters, minimizing the integral gives an exact result. The choice of Gaussian functions as basis functions is preferable, since they exponentially decrease with increasing distance between particles, and at the same time, the matrix elements of such functions can be calculated analytically, provided that the matrix composed of nonlinear parameters $A_{\mathrm{ij}}$ is positive definite. Nevertheless, often such an approximation is not enough and one has to find wave function in the form of a linear combination of basis functions (8), the expansion coefficients of which, up to normalization, are also considered as variational parameters. However, in this case, the number of variable parameters begins to grow rapidly with an increase in the number of basis functions. Nonlinear parameters $A_{\mathrm{ij}}$ are generated as follows:

$$
A_{i j}=\frac{1}{\left(b_{\min }+b_{\max } x\right)^{2}},
$$

where $x$ is a randomly generated number in the interval $(0,1) ; b_{\min }$ and $b_{\max }$ are the set of parameters. In order to fill the interval most compactly in which nonlinear parameters are selected, it is necessary to repeat this procedure a large number of times. The problem is that the overall complexity of solving problems of the form (9), which is associated with the time taken to complete the task, is $\mathrm{O}\left(\mathrm{K}^{3}\right)$, and with an increase in the size of the basis $K$ by a factor of 2 , the computational complexity increases on average by 8 times. This greatly 
slows down the method and makes it difficult to sort through a large number of parameters. To increase the speed of calculation, it is proposed to act in a different way. You can use the results of parameter optimization for smaller bases. Instead of directly solving equation (9) with trial functions (8), an iterative process is constructed, gradually increasing the dimension of the basis from 1 to $K$. At the first iteration, the trial function is represented as one basis. A large set of nonlinear parameters is generated for it. For each of this set, problem (9) is solved and the minimum energy is determined. The set of parameters that gives the least energy is saved. At the next iteration, the dimension of the basis is increased by one, and new sets of parameters are generated only for the new basis function. As a result, at each $n$ iteration, only the parameters of the nth basis function are changed, and for functions successfully found at the previous iterations, only the expansion coefficients are changed. Nevertheless, the growth rate of complexity remains cubic and with large sizes of the basis, the computation speed decreases markedly. In order not to solve problem (9) with completely filled matrices again at each iteration, it is also possible to use the results of calculations obtained at the previous iteration [8].

To start solving (9), it is necessary to know the matrix elements of the Hamiltonian which were calculated by us in previous papers [16-18]. To improve the accuracy of the calculation, it is necessary to consider corrections to the structure of the nucleus and vacuum polarization, relativistic correction. Relativistic corrections, which can be obtained from the Breit Hamiltonian, have the following form in the case of three interacting particles:

$$
V_{B}=-\frac{\alpha^{2}}{8} \sum_{i=1}^{3} \frac{\mathbf{p}_{i}^{4}}{m_{i}^{3}}-\frac{\pi \alpha^{2}}{2} \sum_{\substack{i, j=1 \\ i \neq j}}^{3} e_{i} e_{j}\left(\frac{1}{m_{i}^{2}}+\frac{1}{m_{j}^{2}}\right) \delta\left(\mathbf{r}_{i j}\right)-\frac{\alpha^{2}}{2} \sum_{\substack{i, j=1 \\ i \neq j}}^{3} \frac{e_{i} e_{j}\left(\mathbf{p}_{\mathrm{i}} \mathbf{p}_{\mathrm{j}}+\frac{\mathbf{r}_{\mathrm{ij}}\left(\mathbf{r}_{\mathrm{ij}} \cdot \mathbf{p}_{\mathrm{i}}\right) \mathbf{p}_{\mathrm{j}}}{\mathbf{r}_{\mathrm{ij}}^{2}}\right)}{m_{i} m_{j} r_{i j}},
$$

where $\mathbf{p}$ is momentum operator, $e$ is the charge of particle, $\delta\left(\mathbf{r}_{i j}\right)$ is the delta function. All corrections are presented in electronic atomic units. Correction to the vacuum polarization is determined by

$$
V_{V P}=\frac{\alpha}{3 \pi} \sum_{\substack{i, j=1 \\ i \neq j}}^{3} \frac{e_{i} e_{j}}{r_{i j}} \int_{1}^{\infty} d \zeta \frac{\sqrt{\zeta^{2}-1}\left(2 \zeta^{2}+1\right)}{\zeta^{4}} e^{-2 \gamma \zeta r_{i j}}
$$

where $\gamma=1 / \alpha, \zeta$ is spectral parameter. To calculate corrections (8) - (9) in the case of hyperfine structure, we use the first order of perturbation theory. The general expression for the correction determined by arbitrary potential $\mathrm{V}$ is

$$
\langle V\rangle=\frac{\sum_{i, j=1}^{K} c_{i} c_{j}\left\langle\varphi_{i}|V| \varphi_{j}\right\rangle}{8 \pi^{3}} \sum_{i, j=1}^{K} c_{i} c_{j} \frac{\left.\left(A_{11}^{i}+A_{11}^{j}\right)\left(A_{22}^{i}+A_{22}^{j}\right)-\left(A_{12}^{i}+A_{12}^{j}\right)^{2}\right]^{\frac{3}{2}}}{[}
$$

where $c_{i}, c_{j}$ are linear variation parameters. For the ground state the matrix elements < $\varphi_{i}|V| \varphi_{j}>(8)$ have the following analytical form:

$$
\begin{aligned}
\left\langle\varphi_{i}\left|\mathbf{p}_{1}^{4}\right| \varphi_{j}\right\rangle & =\frac{15(2 \pi)^{3}}{(\operatorname{detB})^{\frac{7}{2}}}\left[\left(A_{11}^{j}+2 \frac{m_{1}}{m_{1}+m_{2}} A_{12}^{j}+\left(\frac{m_{1}}{m_{1}+m_{2}}\right)^{2} A_{22}^{j}\right) \operatorname{det} A^{i}\right. \\
& \left.+\left(A_{11}^{i}+2 \frac{m_{1}}{m_{1}+m_{2}} A_{12}^{i}+\left(\frac{m_{1}}{m_{1}+m_{2}}\right)^{2} A_{22}^{i}\right) \operatorname{det} A^{j}\right]^{2}
\end{aligned}
$$




$$
\begin{aligned}
& \left\langle\varphi_{i}\left|\mathbf{p}_{2}^{4}\right| \varphi_{j}\right\rangle=\frac{15(2 \pi)^{3}}{(\operatorname{det} \mathrm{B})^{7 / 2}}\left[\left(A_{11}^{j}-2 \frac{m_{2}}{m_{1}+m_{2}} A_{12}^{j}+\left(\frac{m_{2}}{m_{1}+m_{2}}\right)^{2} A_{22}^{j}\right) \operatorname{det} A^{i}\right. \\
& \left.+\left(A_{11}^{i}-2 \frac{m_{2}}{m_{1}+m_{2}} A_{12}^{i}+\left(\frac{m_{2}}{m_{1}+m_{2}}\right)^{2} A_{22}^{i}\right) \operatorname{det} A^{j}\right]^{2}, \\
& \left\langle\varphi_{i}\left|p_{3}^{4}\right| \varphi_{j}\right\rangle=\frac{15(2 \pi)^{3}}{(\operatorname{det} \mathrm{B})^{\frac{7}{2}}}\left[A_{22}^{j} \operatorname{det} A^{i}+A_{22}^{i} \operatorname{det} A^{j}\right]^{2}, \\
& \left\langle\varphi_{i}\left|\frac{1}{r_{12}}\left(\mathbf{p}_{1} \mathbf{p}_{2}+\frac{\mathbf{r}_{12}\left(\mathbf{r}_{12} \cdot \mathbf{p}_{1}\right) \mathbf{p}_{2}}{\mathbf{r}_{12}^{2}}\right)\right| \varphi_{j}\right\rangle \\
& =\frac{8(2 \pi)^{5 / 2}}{\sqrt{B_{22}}(\operatorname{detB})^{2}}\left[\left(A_{11}^{i}+\frac{m_{1}-m_{2}}{m_{1}+m_{2}} A_{12}^{i}-\frac{m_{1} m_{2}}{\left(m_{1}+m_{2}\right)^{2}} A_{22}^{i}\right) \operatorname{det} A^{j}\right. \\
& \left.+\left(A_{11}^{j}+\frac{m_{1}-m_{2}}{m_{1}+m_{2}} A_{12}^{j}-\frac{m_{1} m_{2}}{\left(m_{1}+m_{2}\right)^{2}} A_{22}^{j}\right) \operatorname{det} A^{i}\right] \\
& \left\langle\varphi_{i}\left|\frac{1}{r_{13}}\left(\mathbf{p}_{1} \mathbf{p}_{3}+\frac{\mathbf{r}_{13}\left(\mathbf{r}_{13} \cdot \mathbf{p}_{1}\right) \mathbf{p}_{3}}{\mathbf{r}_{13}^{2}}\right)\right| \varphi_{j}\right\rangle \\
& =\frac{8(2 \pi)^{5 / 2}}{(\operatorname{detB})^{2} \sqrt{B_{11}-2 \frac{m_{2}}{m_{1}+m_{2}} B_{12}+\left(\frac{m_{2}}{m_{1}+m_{2}}\right)^{2} B_{22}}} \\
& {\left[\left(\frac{m_{1}}{m_{1}+m_{2}} A_{22}^{i}+A_{12}^{i}\right) \operatorname{det} A^{j}+\left(\frac{m_{1}}{m_{1}+m_{2}} A_{22}^{j}+A_{12}^{j}\right) \operatorname{det} A^{i}\right]} \\
& \left\langle\varphi_{i}\left|\frac{1}{r_{23}}\left(\mathbf{p}_{2} \mathbf{p}_{3}+\frac{\mathbf{r}_{23}\left(\mathbf{r}_{23} \cdot \mathbf{p}_{2}\right) \mathbf{p}_{3}}{\mathbf{r}_{23}^{2}}\right)\right| \varphi_{j}\right\rangle \\
& 8(2 \pi)^{\frac{5}{2}} \\
& =\frac{8(2 \pi)^{\frac{5}{2}}}{(\operatorname{detB})^{2} \sqrt{B_{11}+2 \frac{m_{1}}{m_{1}+m_{2}} B_{12}+\left(\frac{m_{1}}{m_{1}+m_{2}}\right)^{2} B_{22}}} \\
& {\left[\left(\frac{m_{2}}{m_{1}+m_{2}} A_{22}^{i}-A_{12}^{i}\right) \operatorname{det} A^{j}+\left(\frac{m_{2}}{m_{1}+m_{2}} A_{22}^{j}-A_{12}^{j}\right) \operatorname{det} A^{i}\right] .}
\end{aligned}
$$

The matrix elements $\left\langle\delta\left(\mathbf{r}_{i j}\right)>\right.$ of delta-functions also can be calculated directly with the use of (11):

$$
<\delta\left(\mathbf{r}_{12}\right)>=<\delta(\boldsymbol{\rho})>=\iint d \boldsymbol{\rho} d \lambda \delta(\boldsymbol{\rho}) e^{-\frac{1}{2}\left(B_{11} \boldsymbol{\rho}^{2}+B_{22} \lambda^{2}+2 B_{12}(\boldsymbol{\rho} \lambda)\right)}=\frac{(2 \pi)^{\frac{3}{2}}}{\left(B_{22}\right)^{\frac{3}{2}}}
$$

where $B_{22}=A_{22}^{i}+A_{22}^{j}$. When calculating the specific contribution of the matrix element, it is necessary to divide it by the normalization factor. Similar expressions can be obtained for other matrix elements:

$$
\begin{aligned}
& <\delta\left(\mathbf{r}_{13}\right)>=\frac{(2 \pi)^{3 / 2}}{\left(B_{11}-2 B_{12} \frac{m_{2}}{m_{12}}+B_{22}\left(\frac{m_{2}}{m_{12}}\right)^{2}\right)^{3 / 2}}, \\
& <\delta\left(\mathbf{r}_{23}\right)>=\frac{(2 \pi)^{3 / 2}}{\left(B_{11}+2 B_{12} \frac{m_{1}}{m_{12}}+B_{22}\left(\frac{m_{1}}{m_{12}}\right)^{2}\right)^{3 / 2}} .
\end{aligned}
$$

To take into account the correction to the nuclear structure for the ground state of muonic helium we expand the electric form factor as follows:

$$
\left.G_{E}\left(k^{2}\right)\right|_{k^{2} \rightarrow 0}=1-\frac{1}{6} r_{E}^{2} k^{2}
$$


where $r_{E}$ is nuclear charge radius, $k^{2}$ is the photon four-momentum squared. As a result, we obtain the following interaction operator for the correction to the structure of the nucleus in electronic atomic units:

$$
V_{s t r}=-\frac{2 \pi}{3} r_{H e}^{2}\left(e_{H e} e_{\mu} \delta^{3}\left(\mathbf{r}_{12}\right)+e_{H e} e_{e} \delta^{3}\left(\mathbf{r}_{13}\right)\right)
$$

where indexes He, $\mu$ and e represent helion, muon and electron respectively. $r_{H e}$ is the charge radius of helion.

\section{The hyperfine structure of the ground state of three-particle systems}

Consider a system of three charged particles of different masses with spins $S_{1}, S_{2}$ and $S_{3}$. The various possible values of the total spin $\mathbf{S}=\mathbf{S}_{\mathbf{1}}+\mathbf{S}_{\mathbf{2}}+\mathbf{S}_{\mathbf{3}}$ system set the levels of the hyperfine structure of the ground state. For definiteness, we choose all spins equal to $1 / 2$ as in the case of muonic helium-3. In the leading order, the contribution to the hyperfine structure of the spectrum has the following general form:

$$
\begin{gathered}
\Delta E^{h f s}=a<\mathbf{S}_{1} \mathbf{S}_{2}>+b<\mathbf{S}_{1} \boldsymbol{S}_{3}>+c<\mathbf{S}_{2} \mathbf{S}_{3}>, a=\frac{2 \pi \alpha^{2}}{3 m_{p} m_{2}} \frac{\mu_{N}}{S_{1}} \frac{1+\kappa_{2}}{S_{2}}<\delta\left(\mathbf{r}_{12}\right)>, \\
b=\frac{2 \pi \alpha^{2}}{3 m_{p} m_{3}} \frac{\mu_{N}}{S_{1}} \frac{1+\kappa_{3}}{S_{3}}\left\langle\delta\left(\mathbf{r}_{13}\right)>, \quad c=\frac{2 \pi \alpha^{2}}{3 m_{2} m_{3}} \frac{1+\kappa_{2}}{S_{2}} \frac{1+\kappa_{3}}{S_{3}}<\delta\left(\mathbf{r}_{23}\right)>,\right. \text { (30) }
\end{gathered}
$$

where $\kappa_{i}$ is anomalous magnetic moment, $\mu_{N}$ is the magnetic moment of nucleus, $m_{p}$ is the proton mass. After averaging over spin functions [19] and diagonalizing the resulting matrix we obtain the following eigenvalues which determine the hyperfine structure of spectrum:

$$
\begin{gathered}
\lambda_{1}=-\frac{1}{4}(a+b+c)+\frac{1}{2} \sqrt{a^{2}+b^{2}+c^{2}-a b-b c-a c}, \\
\lambda_{2}=-\frac{1}{4}(a+b+c)-\frac{1}{2} \sqrt{a^{2}+b^{2}+c^{2}-a b-b c-a c}, \\
\lambda_{3}=\frac{1}{4}(a+b+c) .
\end{gathered}
$$

Hyperfine structure of ${ }^{4} \mathrm{He} \mu \mathrm{e}$ is determined only by spin-spin interaction of muon and electron. Correction for vacuum polarization to hyperfine splitting has a form:

$$
\begin{gathered}
\Delta E_{V P}^{h f s}=a_{V P}<\mathbf{S}_{1} \mathbf{S}_{2}>+b_{V P}<\mathbf{S}_{1} \boldsymbol{S}_{3}>+c_{V P}<\mathbf{S}_{2} \mathbf{S}_{3}>, \\
a_{V P}=-\left\langle\frac{2 \alpha^{2} g_{1} g_{2}}{3 m_{p} m_{2}} \frac{\alpha}{3 \pi} \int_{1}^{\infty} \rho(\zeta) d \zeta\left[\pi \delta\left(\boldsymbol{r}_{12}\right)-\frac{\gamma^{2} \zeta^{2}}{r_{12}} e^{-2 \gamma \zeta r_{12}}\right]\right\rangle, \\
b_{V P}=\left\langle\frac{2 \alpha^{2} g_{1} g_{3}}{3 m_{p} m_{3}} \frac{\alpha}{3 \pi} \int_{1}^{\infty} \rho(\zeta) d \zeta\left[\pi \delta\left(\boldsymbol{r}_{13}\right)-\frac{\gamma^{2} \zeta^{2}}{r_{13}} e^{-2 \gamma \zeta r_{13}}\right]\right\rangle, \\
c_{V P}=\left\langle\frac{2 \alpha^{2} g_{2} g_{3}}{3 m_{2} m_{3}} \frac{\alpha}{3 \pi} \int_{1}^{\infty} \rho(\zeta) d \zeta\left[\pi \delta\left(\boldsymbol{r}_{23}\right)-\frac{\gamma^{2} \zeta^{2}}{r_{23}} e^{-2 \gamma \zeta r_{23}}\right]\right\rangle .
\end{gathered}
$$

Integration over the Jacobi coordinates can be performed analytically. Thus we obtain the following integral expressions for vacuum polarization correction to the hyperfine structure:

$$
\begin{gathered}
I_{V P, h f s}^{12}=\int_{1}^{\infty} \rho(\zeta) d \zeta\left[\frac{2 \sqrt{2} \pi^{5 / 2}}{B_{22}^{3 / 2}}-\frac{8 \pi^{2} \gamma^{2} \zeta^{2}}{\left(B_{11} B_{22}-B_{12}^{2}\right)^{\frac{3}{2}}} \times\right. \\
\left.\left(\sqrt{2 \pi} \sqrt{B_{11}-\frac{B_{12}^{2}}{B_{22}}}-2 \pi \gamma \zeta e^{\frac{2 B_{22} \gamma^{2} \zeta^{2}}{B_{11} B_{22}-B_{12}^{2}}} \operatorname{erfc}\left(\sqrt{2} \gamma \zeta \sqrt{\frac{B_{22}}{B_{11} B_{22}-B_{12}^{2}}}\right)\right)\right]
\end{gathered}
$$




$$
\begin{aligned}
& I_{V P, h f s}^{13}=\int_{1}^{\infty} \rho(\zeta) d \zeta\left[\frac{2 \sqrt{2} \pi^{\frac{5}{2}}}{\left(F_{1}^{13}\right)^{\frac{3}{2}}}-\frac{8 \pi^{2} \gamma^{2} \zeta^{2}}{\left(F_{1}^{13} B_{22}-\left(F_{2}^{13}\right)^{2}\right)^{\frac{3}{2}}} \times\left(\sqrt{2 \pi} \sqrt{B_{22}-\frac{\left(F_{2}^{13}\right)^{2}}{F_{1}^{13}}}\right.\right. \\
& \left.-2 \pi \gamma \zeta e^{\frac{2 F_{1}^{13} \gamma^{2} \zeta^{2}}{F_{12}^{13} B_{22}-\left(F_{2}^{13}\right)^{2}}} \operatorname{erfc}\left(\sqrt{2} \gamma \zeta \sqrt{\frac{F_{1}^{13}}{F_{1}^{13} B_{22}-\left(F_{2}^{13}\right)^{2}}}\right)\right) \text {. } \\
& I_{V P, h f s}^{23}=\int_{1}^{\infty} \rho(\zeta) d \zeta\left[\frac{2 \sqrt{2} \pi^{\frac{5}{2}}}{\left(F_{1}^{23}\right)^{\frac{3}{2}}}-\frac{8 \pi^{2} \gamma^{2} \zeta^{2}}{\left(F_{1}^{23} B_{22}-\left(F_{2}^{23}\right)^{2}\right)^{\frac{3}{2}}} \times\left(\sqrt{2 \pi} \sqrt{B_{22}-\frac{\left(F_{2}^{23}\right)^{2}}{F_{1}^{23}}}\right.\right. \\
& -2 \pi \gamma \zeta e^{\frac{2 F_{1}^{23} \gamma^{2} \zeta^{2}}{F_{122} B_{22}-\left(F_{2}^{23}\right)^{2}}} \operatorname{erfc}\left(\sqrt{2} \gamma \zeta \sqrt{\left.\left.\frac{F_{1}^{23}}{F_{1}^{23} B_{22}-\left(F_{2}^{23}\right)^{2}}\right)\right)}\right] \text {, } \\
& \Delta E_{V P}^{h f s}=\frac{2 \alpha^{3}}{9 \pi}\left(\frac{g_{1} g_{2}}{m_{p} m_{2}} I_{V P, h f s}^{12} \overline{\left(\mathbf{S}_{1} \mathbf{S}_{2}\right)}+\frac{g_{1} g_{3}}{m_{p} m_{3}} I_{V P, h f s}^{13} \overline{\left(\mathbf{S}_{1} \mathbf{S}_{3}\right)}+\frac{g_{2} g_{3}}{m_{2} m_{3}} I_{V P, h f s}^{23} \overline{\left(\mathbf{S}_{2} \mathbf{S}_{3}\right)}\right),
\end{aligned}
$$

where $\rho(\zeta)=\frac{\sqrt{\zeta^{2}-1}\left(2 \zeta^{2}+1\right)}{\zeta^{4}}, g_{1}, g_{2}$ and $g_{3}$ are gyromagnetic factors of helion, muon and electron, erfc is the complementary error function.

\section{Conclusion}

All our results for the calculation of matrix elements, bound state energy and different corrections to it including hyperfine splitting are presented in Tables 1-3. Using the stochastic variational method we obtain approximate numerical value of the electron Lamb

\begin{tabular}{|c|c|c|c|c|}
\hline & \multicolumn{3}{|c|}{${ }^{3} \mathrm{He \mu e}$} & ${ }^{4} \mathrm{He \mu e}$ \\
\hline & $\delta\left(\boldsymbol{r}_{\mathbf{1 2}}\right)$ & $\delta\left(\boldsymbol{r}_{13}\right)$ & $\delta\left(\boldsymbol{r}_{23}\right)$ & $\delta\left(\boldsymbol{r}_{23}\right)$ \\
\hline Korobov [9] & 20149938.84 & 0.3206115515 & 0.3136823199 & 0.3137605363 \\
\hline Frolov [10] & 20149938.85 & 0.3206118514 & 0.3136818415 & 0.3137605147 \\
\hline Our & 20139692.50 & 0.3204390995 & 0.3137899959 & 0.3138560167 \\
\hline
\end{tabular}
shift $\mathrm{E}(2 \mathrm{P}-2 \mathrm{~S})(\mathrm{He}-3,4)=10 \mathrm{GHz}$. The accuracy of our calculation is not high because of lack of accuracy of obtained matrix elements.

Table 1. Numerical values of the matrix elements of the $\delta$ function in electronic atomic units for the

\begin{tabular}{|c|c|c|}
\hline & ${ }^{3} \mathrm{He \mu e}$ & ${ }^{4} \mathrm{He \mu e}$ \\
\hline $\mathrm{E}, \mathrm{eV}$ & -10858.496122 & -10956.319047 \\
\hline$[9], \mathrm{eV}$ & -10858.495156 & -10956.318081 \\
\hline [10], eV & -10858.495156 & -10956.318081 \\
\hline$E_{B}, e V$ & -0.59457619 & -0.59774665 \\
\hline$E_{\text {str }}, e V$ & 3.1587 & 2.3663 \\
\hline$E_{V P}, e V$ & -17.7806 & -18.4777 \\
\hline$E_{\text {tot }}, e V$ & -10873.702742 & -10973.028228 \\
\hline
\end{tabular}
ground states in the muonic helium.

Table 2. Numerical values of total energy of the ground state, corrections to the structure of the nucleus, relativistic correction, and correction to the vacuum polarization.

The obtained values are consistent with [9, 10]. The existing discrepancies are associated with a smaller basis size in our calculations and, as a consequence, a lower accuracy of the ground state energy. Numerical results depend on the choice of initial parameters, including the masses of particles. The particle mass values in $[9,10]$ are somewhat different from the values recommended by CODATA [20], which we use in the calculations. In $[9,10]$, the matrix elements of the delta functions $a, b$, c, which determine 
the hyperfine splitting, were calculated with very high accuracy, which cannot be achieved within the framework of the stochastic variational method with the basis size used by us. We managed to get a match from $[9,10]$ to five significant digits. It should also be noted that in $[9,10]$, the calculation uses quadruple accuracy, while in our calculations, double precision is used. It also makes a difference in the results.

Table 3. Numerical values of hyperfine splitting with the correction to vacuum polarization.

\begin{tabular}{|c|c|c|}
\hline & ${ }^{3} \mathrm{He \mu e}$ & ${ }^{4} \mathrm{He \mu e}$ \\
\hline $\mathrm{a}, \mathrm{MHz}$ & $3.3166 \cdot 10^{8}$ & - \\
\hline $\mathrm{b}, \mathrm{MHz}$ & 4464.75 & 4465.68 \\
\hline c, $\mathrm{MHz}$ & 1091.09 & - \\
\hline$\lambda_{1}, \mathrm{MHz}$ & $2.48742 \cdot 10^{8}$ & 4465.68 \\
\hline$\lambda_{2}, \mathrm{MHz}$ & $-8.29113 \cdot 10^{7}$ & - \\
\hline$\lambda_{3}, \mathrm{MHz}$ & $-8.29155 \cdot 10^{7}$ & - \\
\hline$\lambda_{1, V P}, \mathrm{MHz}$ & $1.9038 \cdot 10^{4}$ & 0.81 \\
\hline$\lambda_{2, V P}, \mathrm{MHz}$ & $-8.2126 \cdot 10^{4}$ & - \\
\hline$\lambda_{3, V P}, \mathrm{MHz}$ & $-5.4127 \cdot 10^{4}$ & - \\
\hline
\end{tabular}

The work is supported by the Russian Science Foundation (grant 18-12-00128).

\section{References}

1. H. Orth et al, Phys. Rev. Lett. 45, 1483 (1980)

2. M.I. Eides, H. Grotch, and V.A. Shelyuto, Phys. Rep. 342, 62 (2001)

3. A.E. Dorokhov et al., EPJ Web Conf. 204, 05007 (2019)

4. R.N. Faustov et al., EPJ Web Conf. 204, 05005 (2019)

5. V.L. Yakhontov and M.Ya. Amusia, J. Phys. B 27, 3743 (1994)

6. A.A. Krutov and A.P. Martynenko, Phys. Rev. A 86, 052501 (2012)

7. V.I. Korobov, I.V. Puzynin, and S.I. Vinitsky, Phys. Lett. 196 (1987)

8. K. Varga and Y. Suzuki, Comp. Phys. Comm. 106 (1997)

9. D.T. Aznabayev, A.K. Bekbaev and V.I. Korobov, Phys. Part. Nucl. Lett. 15, 3 (2018)

10. A.M. Frolov, Phys. Lett. A 376 (2012)

11. S.D. Lakdawala and P.J. Mohr, Phys. Rev. A 22, 1572 (1980)

12. A.A. Krutov and A.P. Martynenko, Phys. Rev. A 78, 032513 (2008)

13. P. Strasser, K. Shinomura, and H.A. Torii, JPS Conf. Proc. 21, 011045 (2018)

14. S.G. Karshenboim, V.G. Ivanov, and M. Amusia, Phys. Rev. A 91, 032510 (2015)

15. S.G. Karshenboim, V.G. Ivanov, and V.I. Korobov, Phys. Rev. A 97, 022504 (2018)

16. A.V. Eskin, V.I. Korobov, A.P. Martynenko, V.V. Sorokin, EPJ Web of Conf. 204 (2019)

17. A. P. Martynenko, F. A. Martynenko, V. V. Sorokin, O.S. Sukhorukova, A.V. Eskin, Bull. Lebedev Phys. Inst. 46, 4 (2019)

18. V.I. Korobov, A.P. Martynenko, V.V. Sorokin, A.V. Eskin, Phys. Part. Nucl. 50, 5 (2019)

19. L.D. Landau, E.M. Lifshitz, Quantum Mechanics: Non-Relativistic Theory (Pergamon Press, 1965)

20. P.J. Mohr, D.B. Newell, B.N. Taylor, Rev. Mod. Phys. 88, 035009 (2016) 